Technische Universität Ilmenau

Institut für Volkswirtschaftslehre

Diskussionspapier Nr. 64

Wettbewerbliche Auswirkungen eines nichtneutralen Internets

Ralf Dewenter, Thomas Jaschinski und Nadine Wiese

Juli 2009

Institut für Volkswirtschaftslehre

Ehrenbergstraße 29

Ernst-Abbe-Zentrum

D-98 684 Ilmenau

Telefon 03677/69-4030/-4032

Fax 03677/69-4203

http://www.wirtschaft.tu-ilmenau.de

ISSN 0949-3859 


\title{
Wettbewerbliche Auswirkungen eines nichtneutralen Internets
}

\author{
Ralf Dewenter, Thomas Jaschinski und Nadine Wiese
}

Technische Universität Ilmenau, Fachgebiet Wirtschaftstheorie, Ehrenbergstraße 29, 98693 Ilmenau

\section{Einleitung}

In den letzten Monaten und Jahren wird insbesondere in den USA aber auch in Europa eine intensive Diskussion darüber geführt, inwiefern die bisher praktizierte Gleichbehandlung der Datenpakete im Internet regulatorisch festgeschrieben werden sollte (vgl. z.B. Hahn \& Wallsten, 2006; Sidak, 2006; van Schewick, 2007; Litan \& Singer, 2007). Die sogenannte Netzneutralitätsdebatte kam vor allem deshalb auf, weil einige Breitbandnetzbetrieber, wie z.B. AT\&T, Verizon und Comcast, planten, Inhalteanbieter, wie Google oder Amazon, für die Durchleitung deren Inhalte zu den Endkunden stärker und abhängig von bestimmten Eigenschaften der zu transportierenden Daten zu bepreisen (vgl. z.B. Cheng, Bandyopadhyay \& Guo, 2007). Ermöglicht wurde dieses Vorhaben erst durch technische Innovationen, die dazu geführt haben, dass Informationen über die versendeten Datenpakete, wie deren Ursprung oder Ziel, der Inhalt oder Verwendungszweck, quasi ohne Zeitverlust und auch bei großem Datendurchsatz ausgelesen und analysiert werden können. Auf diese Weise kann nun problemlos zwischen verschiedenen Diensten, Volumen oder Inhalten diskriminiert werden. Mögliche Anwendungen dieser Technik lägen neben einer stärker differenzierten Bepreisung von Inhalteanbietern und Endkunden unter anderem in der Möglichkeit, eine Priorisierung einzelner Datenpakete vorzunehmen, einzelne Inhalte vom Netz auszuschließen oder generell darin, eine stärkere Form der Preis- oder Qualitätsdifferenzierung zu betreiben.

Gegen dieses Vorhaben haben sich nicht nur die von einem Preisanstieg direkt betroffenen Inhalteanbeiter ausgesprochen. Es existiert darüber hinaus auch eine Gruppe von Befürwortern der Netzneutralität, die vor allem negative Auswirkungen dieser neuen Technik befürchten, wie zum Beispiel den Ausschluss von Wettbewerbern oder die Anwendung einer wohlfahrtsreduzierenden Diskriminierung. Das vorliegende Papier diskutiert daher die möglichen negativen wettbewerblichen und wohlfahrtsökonomischen Auswirkungen eines nichtneutralen Internets und 
stellt kurz den Stand der Diskussion zu den wichtigsten ökonomischen Aspekten dar. Ziel ist es, einen Überblick über die zu erwartenden Effekte zu geben und darüber hinaus eine Einschätzung über die Notwendigkeit einer Netzneutralitätsregulierung - also einer regulatorisch festgeschriebenen Neutralität - abzugeben.

Das Papier ist wie folgt aufgebaut. Zunächst werden in Kapitel 2 die Struktur des Internets sowie die wichtigsten Marktteilnehmer dargestellt. In Kapitel 3 erfolgt dann eine Analyse der statischen und dynamischen Auswirkungen eines nichtneutralen Internets bezüglich verschiedener Formen der Diskriminierung und Differenzierung sowie der Innovationswirkungen. Kapitel 4 fasst dann noch einmal die wichtigsten Ergebnisse zusammen und schließt mit einem Fazit.

\section{Die Struktur des Internets}

Die ursprüngliche Architektur des Internets stellt sich als nichtintelligentes Netzwerk dar, das keine Unterscheidung einzelner Datenpakete vornehmen konnte. Grundlage für die Versendung der Daten ist dabei die sog. Besteffort-rule. Werden Datenpakete gemäß dieser Regel versendet, die besagt, dass die Übermittlung unter Ausnutzung der verfügbaren Kapazitäten schnellstmöglich vorgenommen werden soll, so werden die Daten weder nach Inhalt noch nach anderen Kriterien diskriminiert. Vielmehr werden sie gleichberechtigt versendet. Die Übermittlung einzelner Datenpakete im Internet wird von der Computerhardware, genauer gesagt von den Routern, übernommen. Sie sind sowohl für die Organisation der Übertragungswege der zu übertragenden Daten als auch für deren Übermittlung verantwortlich (vgl. Ganley \& Allgrove, 2006). Auch im Fall einer Überlast des Netzes werden die beim Router eintreffenden Pakete nach dem First-In/First-Out Prinzip behandelt. Die Übermittlung der Daten und Inhalte kann somit als neutral und nichtdiskriminierend bezeichnet werden.

Technische Innovationen (so etwa die Service Control Technology von Cisco aus dem Jahr 1999) ermöglichen inzwischen jedoch eine unterschiedliche Behandlung von Datenpaketen. Auf diese Weise können zum Beispiel nach einer Inspektion der Inhalte und einer Bewertung einer zuvor zugewiesenen Priorität (,access tiering“) Daten gezielt diskriminiert werden. Anwendungen, die keiner besonderen Priorität bedürfen, wie Emailing oder verzögerungsunkritische Internetanwendungen, könnten dabei nachrangig behandelt werden. VoIP und Videoanwendungen hingegen könnten eine bevorzugte Behandlung erfahren. Eine stärkere Verwendung der technologischen Innovationen lässt also vor allem eine Differenzierung hinsichtlich der Qualität und Eigenschaften der Dienste zu. 
Betroffen von diesen Innovationen sind zum einen die Endkunden, die die verschiedenen Dienste und Angebote wahrnehmen und zum anderen natürlich die Internetdienstleister. Die im Zusammenhang mit der Netzneutralität relevanten Dienstleister sind dabei die Carrier, die sog. Content Provider (CP) und Internet Service Provider (ISP).

Als Carrier werden dabei die Unternehmen bezeichnet, die die zur Übertragung des Datenverkehrs notwendigen Teilnetze betreiben. Diese Teilnetze stellen die Hauptverbindungen bzw. die Infrastruktur des Internets dar. Carrier sind also Netzbetreiber und bieten damit Übertragungsleistungen für andere Unternehmen an. Mit Content Provider (auch Inhalteanbieter oder Application Provider) werden dagegen Anbieter von Inhalten verschiedenster Form - so z.B. Nachrichten- und Informationsplattformen oder Anbieter von Applikationen, wie etwa Suchmaschinen oder Auktionsplattformen - bezeichnet. Anbieter, die als Access Provider, oder gewöhnlich auch als Internet Service Provider, bezeichnet werden, ermöglichen Internet-Nutzern den Zugang zum Internet, d.h. bieten Internet-Konnektivität an. In aller Regel sind ISPs, wie z.B. Deutsche Telekom, vertikal integriert, d.h. sie treten regelmäßig auch als Carrier und/oder Content Provider auf.

Bemerkenswert ist dabei, dass sowohl Carrier als auch ISPs und CPs typischerweise einer besonderen Nachfragestruktur gegenüberstehen. So treten die meisten Provider nicht nur als Anbieter verschiedener Dienste auf, sondern agieren gleichzeitig als sogenannte zweiseitige Plattformen (vgl. Rochet \& Tirole, 2003, zur Theorie der zweiseitigen Märkte und Dewenter, 2008, oder Economides \& Tåg, 2008, zu Service Providern als zweiseitige Plattformen). So bieten Content Provider zum einen ihre Inhalte am Rezipientenmarkt an. Zum anderen verkaufen sie (sofern sie werbefinanziert sind) die Aufmerksamkeit der Rezipienten an die Werbekunden. Zwischen diesen beiden Nachfragergruppen liegen aber typischerweise indirekte Netzwerkeffekte vor, sodass der Nutzen der einen Gruppe durch die Größe der jeweils anderen Gruppe beeinflusst wird. Dies führt $\mathrm{zu}$ einer komplementären Abhängigkeit der Nachfrage, was wiederum direkte Auswirkung auf die Preisgestaltung und das strategische Verhalten der Provider nimmt. Eine Veränderung der Preise, der Qualitäten oder der Produktdifferenzierung betrifft somit immer auch den jeweils verbundenen Markt. Die Anreize, z.B. die Preise für einen bestimmten Dienst zu erhöhen, sind daher tendenziell geringer. Ähnliche Beziehungen lassen sich auch für ISPs und Carrier darstellen. So vermitteln Carrier zwischen den CPs auf der einen und den ISPs auf der anderen Seite. ISPs hingegen stellen eine Plattform dar, die zwischen den Rezipienten und den Carriern (bzw. den CPs) vermitteln. Eine ausführliche Analyse der Auswirkungen der Netzneutralität muss daher auch immer im Kontext der zweiseitigen Plattformen (bzw. Märkte) vorgenommen werden. 


\section{Auswirkungen der Aufhebung von Netzneutralität}

Die typischen Auswirkungen eines nichtneutralen Internets lassen sich grob in die Kategorien Ausschluss, Qualitätsdifferenzierung durch Quality of Service, Preisdiskriminierung und Innovationswirkungen unterteilen. Alle vier Kategorien werden im Folgenden einer näheren Betrachtung unterzogen und bezüglich der wettbewerblichen Wirkungen analysiert.

\subsection{Ausschluss}

Ein häufig genanntes Argument der Befürworter von Netzneutralität ist die Befürchtung, bestimmte Angebote oder Nutzer könnten vom Internet bzw. von Teilen des Netzes ausgeschlossen werden. Insbesondere ISPs, so die Vermutung, könnten Anreize haben, ein solches Blocking von Inhalten durchzuführen und damit wettbewerbsbeschränkende sowie wohlfahrtsmindernde Effekte hervorrufen.

Unter Blocking werden im Allgemeinen die Maßnahmen verstanden, die den Zugang zu bestimmten Inhalten oder Internetseiten beschränken. Ein typisches Beispiel dafür ist, dass Internet Service Provider Inhalte mit bestimmten Eigenschaften (z.B. Viren, Spammails etc.) vom eigenen Netz ausschließen, diese Daten also nicht transportieren. Solange Daten geblockt werden, die einen Disnutzen erzeugen, geht kein negativer Effekt vom Blocking aus. Werden von den ISPs aber kategorisch bestimmte Inhalte oder komplette Seiten der Inhalteanbieter geblockt und somit das Angebot am Content Markt reduziert, entsteht dann ein Wohlfahrtsverlust, wenn eine Zahlungsbereitschaft für diese Inhalte (entweder über eine Werbefinanzierung oder ein Pay-Angebot) vorhanden ist. Ein Anreiz zu einem derartigen Blocking könnte vor allem für vertikal integrierte ISPs bestehen (vgl. Dewenter, 2008). Unter Umständen kann es für einen ISP, der gleichzeitig auch bestimmte Inhalte anbietet, gewinnmaximierend sein, andere Anbieter, die am Inhaltemarkt mit dem ISP konkurrieren, vom Netz auszuschließen, um dadurch den Wettbewerb auszuschalten.

Ein solcher Ausschluss könnte insbesondere dann profitabel sein, wenn die entsprechenden vertikal integrierten ISPs Marktmacht aufweisen oder gar als vertikal integrierter Monopolist auftreten. ${ }^{1}$ Auch wenn eine solche Situation nicht unbedingt typisch für Internetdienste ist, lässt sie sich a priori nicht ganz ausschließen. Im Falle eines solchen wohlfahrtsmindernden Blockings würden aber sowohl nach deutschem ${ }^{2}$ als

\footnotetext{
${ }^{1}$ Für eine ausführliche Diskussion möglicher Anreize vgl. z.B. van Schewick (2007) oder Dewenter (2008).

${ }^{2} \S 19$ GWB - „Missbrauch einer marktbeherrschenden Stellung“ - gilt insbesondere für die Weigerung, Zugang zu Netzen und Infrastruktureinrichtungen zu gewähren.
} 
auch nach europäischem ${ }^{3}$ und US-amerikanischem ${ }^{4}$ Wettbewerbsrecht die Regulierung einer wesentlichen Einrichtung („Essential Facility Doktrin“) und die Missbrauchsaufsicht greifen. Diese Institutionen dienen als angemessene Mittel, im Falle marktbeherrschender ISPs, wettbewerbsschädliche Diskriminierung bzw. Blocking zu untersagen (vgl. auch Vogelsang, 2007).

Anders könnte sich die Situation möglicherweise in Oligopolmärkten darstellen, in denen scheinbar keine Marktbeherrschung vorliegt. Van Schewick (2007) z.B. führt für diesen Fall vier Beispiele an, in denen Anreize zum Ausschluss vorliegen könnten:

1. Im Falle von Pay-Inhalten erhöht ein Ausschluss konkurrierender Inhalte die Nachfrage nach eigenen Inhalten und führt bei sinkenden Durchschnittskosten zu einem Anstieg des Gewinns.

2. Ein Ausschluss konkurrierender Inhalte erhöht die Umsätze aus der Werbung im Content Markt.

3. Ein Ausschluss konkurrierender Inhalte gewährleistet die Monopolstellung im nationalen Content Markt.

4. Wenn ein ISP seinen Content nur seinen eigenen Kunden anbietet, kann ein Ausschluss konkurrierender Inhalte die oligopolistische Stellung am Markt für Internetzugänge sichern.

Allen vier Beispielen ist gemein, dass die Höhe der Wechselkosten, die dem Nutzer beim Wechsel des ISP entstehen, ausschlaggebend dafür ist, ob tatsächlich Anreize bestehen, CPs vom Netz auszuschließen. Eine Reduzierung der (Qualität der) Inhalte mindert den Nutzen der Rezipienten, allerdings könnten diese mit einem Wechsel des ISP reagieren. Je höher aber die Wechselkosten sind, desto weniger wechselwillige Kunden sind kurzfristig in der Lage, zu einem konkurrierenden ISP zu wechseln. Mittelbis langfristig sollte dieses Problem aber nicht evident und ein Wechsel des ISP möglich sein. Hinzu kommt, dass der ISP bei seinen Blocking-Anreizen berücksichtigen muss, dass er im Falle von Blocking auf die Erlöse verzichtet, die durch den Verkehr der geblockten Inhalte auf seinem Netz generiert werden könnten.

\footnotetext{
${ }^{3}$ Art. 82 EGV verbietet ebenso die Ausnutzung einer marktbeherrschenden Stellung.

${ }^{4}$ Hier kann der verpflichtende Zugang zu wesentlichen Einrichtungen gemäß sec. 2 des Sherman Acts und der Essential Facility Doktrin begründet werden.
} 
In den Beispielen 1 und 2 besteht also nur dann ein Anreiz zum Blocking, wenn die Erlöse der durch Blocking gewonnenen Nachfrage diejenigen Erlöse übersteigt, die durch den Verkehr der nun geblockten Inhalte auf dem Netz des ISP zusätzlich hätten generiert werden können. Im Falle werbefinanzierter Inhalte (Beispiel 2) ist außerdem zu berücksichtigen, dass nicht nur die Inhalte geblockt werden müssten, die Substitute für die Nutzer darstellen, sondern ebenso Inhalte, die mögliche Substitute für die Werbekunden darstellen. Mehr noch als im Monopolfall sind oligopolistische ISPs aber darauf angewiesen, den Internet-Nutzern den Zugriff auf ein möglichst großes Angebot an Inhalten zu ermöglichen, um eine Abwanderung $\mathrm{zu}$ einem anderen ISP zu verhindern und somit die Nachfrage am oligopolistischen Accessmarkt zu sichern. Ein Ausschluss verschiedener Inhalte, zudem noch solcher Inhalte, die eine große Nachfrage bei den Endkunden aufweisen, scheint daher unwahrscheinlich.

Beispiel 3 diskutiert die Möglichkeit für oligopolistische ISPs, den gesamten nationalen Content Markt durch Blocking konkurrierender Inhalte an einem regionalen Markt zu monopolisieren. Eine erfolgreiche Monopolisierung durch Blocking ist dabei aber $\mathrm{zu}$ bezweifeln. Voraussetzung dafür wäre die Existenz extrem starker Netzwerkeffekte (bzw. ein sehr großer regionaler Markt) und einer starken Marktmacht am Accessmarkt (vgl. Dewenter, 2008). Liegt eine solche marktmächtige Stellung des ISP jedoch vor, würde die Missbrauchsaufsicht greifen. Existiert hingegen Wettbewerb am Accessmarkt, könnten die geblockten Inhalte von konkurrierenden ISPs angeboten werden.

Beispiel 4 unterstellt, dass ISPs einen Anreiz haben könnten, konkurrierende Inhalte zu blocken, wenn die eigenen Inhalte auf die eigenen Kunden beschränkt sind. Bestimmte Inhalte wären dann nur bei einem bestimmten ISP verfügbar, sodass eine stärkere Produktdifferenzierung zu anderen ISPs vorliegt. Daraus folgen für die ISPs sowohl ein weniger starker Preiswettbewerb als auch eine Erhöhung der Wechselkosten der Nutzer, da besagte Inhalte den Kunden bei einem Wechsel zu einem anderen ISP nicht mehr zur Verfügung stehen. Aufgrund der höheren Wechselkosten ist hier von einem größeren negativen Wohlfahrtseffekt auszugehen als bei den anderen Beispielen. Mittel- bis langfristig sollte die Höhe der Wechselkosten allerdings an Bedeutung verlieren, wenn wechselwillige Kunden jederzeit in der Lage sind, die Inhalte und Geschäftsbedingungen verschiedener ISPs zu vergleichen.

Zusammenfassend kann festgehalten werden, dass auch für oligopolistische Märkte keine überzeugenden Argumente dafür existieren, dass ISPs Anreize hätten, Konkurrenten am Inhaltemarkt vom Netz auszuschließen. Voraussetzung ist regelmäßig eine gewisse Marktmacht. Liegt jedoch Marktmacht vor, so ist eine Untersagung des Blockings nach dem Wettbewerbsrecht möglich. 


\subsection{Qualitätsdifferenzierung durch Quality of Service}

Der Verzicht auf eine Regulierung der Netzneutralität würde des Weiteren eine unterschiedliche Behandlung bezüglich der Übermittlungsqualität (Quality of Service, QoS) ermöglichen. Dadurch könnten die einzelnen Pakete analysiert und ihnen verschiedene Prioritäten zugewiesen werden. Dies könnte dann in Form einer bevorzugten Weiterleitung unterschiedliche Servicequalitäten zur Folge haben.

Im Falle einer Regulierung der Netzneutralität wären die Netzbetreiber dazu gezwungen, allen Nachfragern eine einheitliche - somit mittlere - Qualität anzubieten. Ein Teil der Konsumenten hat jedoch keine Zahlungsbereitschaft für diese mittlere Qualität, der Konsum würde ausbleiben. Für einen anderen Teil der Konsumenten ist die angebotene Qualität niedriger als die nachgefragte Qualität, ihre Nachfrage nach hoher Qualität bleibt unerreicht. Somit existiert eine Situation allokativer Ineffizienz. Mit Hilfe einer Differenzierung der Qualität können anstatt nur einer ineffizienten mittleren Qualität unterschiedliche Qualitäten angeboten werden, die je nach Zahlungsbereitschaft der Nachfrager ihren Präferenzen entsprechen (vgl. dazu Kruse, 2009, in diesem Band; vgl. auch Litan \& Singer, 2007).

Ein ebenso großer Vorteil einer Qualitätsdifferenzierung ist, dass bei einer hohen Auslastung der Netze und insbesondere der Router, unterschiedliche Inhalte und Anwendungen ihren Bedürfnissen entsprechend priorisiert werden können (,access tiering“). Damit kann für Daten mit hoher zugewiesener Priorität zumindest im Vergleich zu anderen Datenpaketen immer eine gewisse Mindestqualität erreicht werden. ${ }^{5} \mathrm{Mit}$ Hilfe des access tiering kann der Nutzen derjenigen Anwender erhöht werden, die besonders zeit- bzw. qualitätssensitive Dienste (VoIP, OnlineSpiele, File-Sharing Dienste, IP-TV etc.) beanspruchen, ohne den Nutzen der Anwender von Emailing oder Webbrowsing stark zu beeinträchtigen. Eine angemessene Bepreisung der unterschiedlichen Prioritäten gemäß den Zahlungsbereitschaften könnte somit prinzipiell das Stauproblem bei Überlast der Router lösen (vgl. Kruse, 2007 und Kruse, 2009).

Neben den positiven Effekten von QoS lassen sich aber auch mögliche negative Auswirkungen identifizieren. Economides (2008) z.B. zeigt eine mögliche Situation auf, in der Wettbewerb durch eine Qualitätsdifferenzierung beschränkt werden könnte. So könnte beispielsweise ein nicht vertikal integrierter monopolistischer ISP einen ausgewählten CP kostenlos priorisieren, was einen relativen Qualitätsverlust für alle anderen CPs zur Folge hätte. Die benachteiligten CPs wären nun

\footnotetext{
${ }^{5}$ Eine absolute Mindestqualität kann dagegen nicht gewährleistet werden, da eine mögliche Überlast dazu führen könnte, dass diese nicht erreicht werden kann. Lediglich eine Bevorzugung gegenüber anderen Daten ist hier möglich.
} 
dazu genötigt, hohe Preise für eine ebenso hohe Übertragungsqualität zu zahlen. Somit wäre der ISP in der Lage, einen Großteil der Gewinne der CPs zu extrahieren. Herrscht jedoch (auch nur geringer) Wettbewerb im Markt für ISPs, kann ein benachteiligter CP zu einem anderen ISP wechseln. Letztendlich gilt jedoch auch hier, dass marktbeherrschende Unternehmen sowohl in Deutschland als auch in Europa und den USA der Missbrauchsaufsicht unterliegen.

Darüber hinaus weist van Schewick (2007) auf einen möglichen Anreiz eines oligopolistischen und vertikal integrierten ISP hin, die Servicequalität, d.h. die Übertragungsqualität, seiner konkurrierenden CPs zu verringern. Eine geringe Übertragungsqualität der CPs wäre von den Konsumenten aufgrund der Vielzahl möglicher Ursachen für die schlechtere Qualität nicht unmittelbar auf eine Diskriminierung durch den ISP zurückzuführen. Die Inhalte des ISP würden dann bevorzugt nachgefragt und seine Gewinne damit maximiert werden. Werden aber in einem nichtneutralen Internet unterschiedliche Qualitäten unterschiedlich bepreist, kann davon ausgegangen werden, dass Übertragungsqualitäten regelmäßig überprüft werden. Somit wäre eine solche Diskriminierung nicht denkbar.

Insgesamt lässt sich feststellen, dass die Anwendung einer Qualitätsdifferenzierung zu positiven Wohlfahrtseffekten führen würde. Mögliche wettbewerbsschädliche Verhaltensweisen bei Vorliegen einer marktbeherrschenden Stellung werden durch die Missbrauchsaufsicht abgedeckt. Bei Vorliegen von wettbewerblichen (z.B. oligopolistischen) Marktstrukturen sollte eine Überprüfung der vertraglich vereinbarten Qualitäten technisch durchführbar sein.

\subsection{Preisdiskriminierung}

Die Aufhebung der Netzneutralität würde ebenso dazu führen, dass eine unterschiedliche Bepreisung sowohl bezüglich einer unterschiedlichen Preishöhe als auch bezüglich einer unterschiedlichen Preisstruktur durchgeführt werden könnte. So ist vorstellbar, dass Carrier oder ISPs solche Content Provider, die ein großes Datenvolumen generieren oder eine höhere Zahlungsbereitschaft aufweisen, nach einem anderen System bepreisen als andere CPs.

Die Durchführung von Preisdiskriminierung bei Inhalten und Anwendungen ist aus ökonomischer Sicht prinzipiell als positiv zu bewerten, wenn dadurch mit einer Steigerung der Wohlfahrt zu rechnen ist (vgl. Edgeworth, 1927; Pigou, 1938; Hausman \& MacKie-Mason, 1988 oder Varian, 1988). Als wohlfahrtökonomisch positiv zu bewerten sind insbesondere Situationen, in denen eine Konsumentengruppe aufgrund ihrer unterdurchschnittlichen Zahlungsbereitschaft erst durch Preisdiskrimi- 
nierung ein Gut nachfragt und der Konsum ansonsten ausgeblieben wäre. Die Ramsey-Regel (vgl. Ramsey, 1927) zeigt, dass eine Bepreisung gemäß den inversen Nachfrageelastizitäten für einzelne Dienste unter der Nebenbedingung der Eigenwirtschaftlichkeit zu einem Second-best Ergebnis und somit zu einer Maximierung der Wohlfahrt führen kann. Es erscheint also nicht ersichtlich, warum eine Preisdiskriminierung für Endkunden oder auch Content Provider untersagt werden sollte.

Mögliche negative Auswirkungen von Preisdiskriminierung werden von Economides (2008) diskutiert. Der Autor stellt dabei eine Situation dar, in der die Durchführung von Preisdifferenzierung eines monopolistischen ISP wohlfahrtsreduzierende Wirkung hat. In seiner Analyse werden jedoch vor- und nachgelagerte Märkte nicht beachtet. Aufgrund dieser Unvollständigkeit lässt sich die Analyse als ökonomisch nicht relevant bewerten. Denn insbesondere in zweiseitigen Märkten führt Preisdifferenzierung in der Regel zu effizienten Ergebnissen statt zu einem Wohlfahrtsverlust (vgl. Dewenter, 2008).

Zusammenfassend kann festgestellt werden, dass Preisdifferenzierung in der Regel als wohlfahrtsökonomisch positiv zu bewerten ist. Negative Auswirkungen sind allenfalls dann $\mathrm{zu}$ erwarten, wenn marktmächtige Unternehmen Nachfrager dazu zwingen können, differenzierte Preise zu akzeptieren. Dann würde allerdings das deutsche bzw. das europäische und US-amerikanische Wettbewerbsrecht greifen und das Verhalten untersagen.

\subsection{Innovationswirkungen}

Neben den direkten Wirkungen auf das wettbewerbliche Verhalten der Marktteilnehmer ist zu vermuten, dass die (Nicht-)Neutralität des Internets ebenso Auswirkungen auf das Innovationsverhalten und damit auf die dynamische Effizienz nimmt.

Generell sind hier zwei unterschiedliche Wirkungen bezüglich der Innovationen unter den beiden Regimes zu erwarten. Da unter Netzneutralität ein nichtintelligentes Netzwerk existiert, also keine Analyse und Diskriminierung der Daten vorgenommen werden kann, sind Innovationen bezüglich der Infrastruktur nur schwer möglich. Innovationen in die Netzintelligenz werden damit unterbunden. Stattdessen werden Innovationen eher an den Rändern der Netze gefördert. Wenn die Intelligenz der Netze also nicht gefördert werden kann, existiert ein Anreiz, die Innovationen im Bereich der Anwendungen oder auch der Hardware, die nicht mehr zum Netz gehört (Endgeräte), zu schaffen.

Unter dem Regime der Nichtneutralität sind grundsätzlich Innovationen in beiden Bereichen möglich. So könnten weiterhin neue Produkte und Verfahren im Bereich der Endgeräte und Anwendungen 
geschaffen werden. Darüber hinaus ließe sich jedoch das „dumme“ Netz in ein intelligentes Netz überführen, sodass auch hier Innovationen eingeführt werden können. Neben den bereits genannten Neuerungen bezüglich der Sicherheit oder der Servicequalität bzw. der Priorisierung, ist hier eine Reihe unterschiedlichster Produkt- und Prozessentwicklungen denkbar.

Zwar ist es unter Nichtneutralität grundsätzlich möglich, in beiden Bereichen - Netz und Anwendungen - zu innovieren, unklar ist jedoch, wie stark die Innovationsanreize bezüglich der Peripherie und der Anwendungen tatsächlich sind. Befürworter der Neutralität argumentieren, dass es bei Nichtneutralität zwar zu einem Anstieg der Innovationen der ISPs im Bereich der Anwendungen kommen würde, zum anderen jedoch eine deutliche Reduktion der Innovationstätigkeit der unabhängigen Content Provider zu befürchten wäre. Weiterhin wird ausgeführt, dass der Anstieg der innovativen Tätigkeit der ISPs nicht ausreicht, um den allgemeinen Rückgang an Neuerungen zu kompensieren (vgl. z.B. van Schewick, 2007). Begründet wird diese Behauptung damit, dass (i) ein Ausschluss von Content Providern ausgehend von vertikal integrierten ISPs die Gewinne der CPs reduziert und damit ein geringerer Anreiz zur Innovation entsteht, dass (ii) die Unsicherheit darüber, ob der ISP einen innovativen CP vom Netz ausschließen wird, die Anreize vermindert und, dass (iii) nicht vertikal integrierte ISPs einen Anreiz haben, erfolgreiche Innovationen zu imitieren und dann ihren Vorteil nutzen, um den größten Teil des Sekundärmarktes für sich zu beanspruchen. Ein innovativer CP würde dieses Ergebnis antizipieren und somit erst gar nicht in den Sekundärmarkt innovieren.

Gegen diese Argumentation ist zunächst einzuwenden, dass ein Ausschluss von Wettbewerbern im Sekundärmarkt, wie vorher schon diskutiert, nicht möglich ist, solange eine Marktbeherrschung vorliegt. Innovative Content Provider könnten in dem Fall die Missbrauchsaufsicht anrufen, um ein mögliches Blocking zu verhindern. Weiterhin zeigen Farrell und Katz (2000), dass ein innovierendes Unternehmen im komplementären (Sekundär-)Markt einen First-Mover-Vorteil hat und entweder die gesamten Gewinne oder zumindest die Hälfte aller Innovationsgewinne realisieren kann. Dies gilt dann, wenn beide Produkte (hier Netz und Anwendung) essentiell für das jeweilige andere Produkt ist und gleiche Kostenstrukturen herrschen. Casadesus-Masanell, Nalebuff \& Yoffee (2006) zeigen, dass eine paritätische Aufteilung der Gewinne auch für asymmetrische Kostenstrukturen gilt. Wie Chen und Nalebuff (2006) zeigen, kann ein unabhängiger Content Provider mehr als die Hälfte der Gewinne für sich beanspruchen, wenn ein Gut (hier die Anwendung) nicht essentiell für die Bereitstellung des anderen Gutes (das Netz) ist. Ein Innovationsanreiz wäre, wenn auch eventuell zu geringeren Gewinnen als unter Netzneutralität, durchaus gegeben. 
Solange Innovationen am Rand des Netzes aber noch realisiert werden, haben auch vertikal integrierte ISPs entweder einen Anreiz, ebenfalls zu innovieren oder aber einen Teil der Innovationsgewinne zu extrahieren. Im Gegensatz zu unabhängigen CPs können vertikal integrierte ISPs die Netzwerkeffekte zwischen Primär- und Sekundärmarkt zumindest zu einem Teil internalisieren (vgl. Kocsis \& de Bijl, 2007). Der ISP hat demnach also einen größeren Anreiz, sowohl im Primärmarkt als auch im Sekundärmarkt zu innovieren. Die Frage ist also nicht, ob Content Provider vom Netz ausgeschlossen werden, sondern eher die nach der Höhe der Gewinne, die innovative CPs extrahieren können, wie stark Innovationen von unabhängigen CPs (aufgrund geringerer erwarteter Gewinne aus Innovationen) zurückgehen würden und in welchem Verhältnis dieser Rückgang zu den zusätzlichen Innovationen der ISPs - am Rand des Netzes und in die Netzintelligenz - stehen würde. Diese Frage ist aufgrund der Komplexität der Marktstrukturen nur schwer zu beantworten. Allerdings darf nicht vergessen werden, dass aufgrund der Mehrseitigkeit der Plattformen und damit aufgrund der vorhandenen direkten und indirekten Netzwerkexternalitäten letztendlich immer der Wettbewerb um die Aufmerksamkeit der Konsumenten ausschlaggebend für den Erfolg einer Anwendung aber auch für den Erfolg des Infrastrukturanbieters ist.

Insgesamt kommt die bisherige Literatur, die Innovationswirkungen der Netzneutralität untersucht, zu ambivalenten Ergebnissen. Insgesamt lässt sich aber feststellen, dass ein Großteil der Analysen eher negative Wirkungen der Netzneutralität konstatiert (vgl. Tabelle 1). So berichten lediglich Cañón (2009) und Choi \& Kim (2008) und Economides (2008) von ausschließlich positiven Effekten eines neutralen Internets. Van Schewick (2007) konstatiert dagegen den bereits erwähnten negativen Effekt auf den Kern des Netzes und einen positiven Effekt auf den Randbereich.

Viele andere Autoren kommen dagegen zum Schluss, dass sowohl im Kern des Netzes als auch am Rand die Innovationen zunehmen würden, wenn von Netzneutralität abgesehen würde. Sidak (2006) z.B. bezieht sich bei seiner Argumentation auf die beobachtbaren Auswirkungen der Deregulierung des Telekommunikationssektors auf die Innovationstätigkeit der Unternehmen. Er interpretiert den Anstieg der Investitionen sowohl in die Infrastruktur als auch im Applikationsbereich dahingehend, dass Regulierung im Allgemeinen innovationshemmend wirkt und eine derartige Wirkung auch für die regulative Festschreibung der Netzneutralität zu befürchten ist. Ähnliche Überlegung findet sich auch bei Owen (2007) und Litan \& Singer (2007).

Eine Reihe von Autoren (vgl. z.B. Hermalin \& Katz, 2007; Renda, 2008; Jamison \& Hauge, 2008; und Musacchio, Schwartz \& Walrand, 2009) wendet die Theorie der zweiseitigen Märkte auf das Problem der 
Innovationen im Internet an und kommen in aller Regel zu dem Ergebnis, dass Nichtneutralität die Innovationstätigkeit fördert, da grob gesagt ein Ausnutzen der Netzwerkeffekte höhere Gewinne verspricht und dadurch Innovationen lohnender sind. Andere Autoren, wie z.B. Cañón (2009) oder Choi \& Kim (2008), kommen zum Schluss, dass Netzneutralität durchaus auch positive Auswirkungen auf die Innovationstätigkeit haben kann. Insgesamt liegt aber keine klare Evidenz für die Notwendigkeit der Regulierung von Netzneutralität vor.

Tabelle 1: Netzneutralität und Innovationen/Investitionen

\begin{tabular}{|c|c|c|c|c|}
\hline & \multirow[b]{2}{*}{ Ansatz } & \multicolumn{3}{|c|}{$\begin{array}{l}\text { Innovationseffekte der } \\
\text { Netzneutralität }\end{array}$} \\
\hline & & Kern & Rand & Gesamt \\
\hline Cañón (2009) & $\begin{array}{l}\text { modellgestützt, zweiseitige } \\
\text { Märkte }\end{array}$ & + & k.A. & + \\
\hline Choi \& Kim (2008) & $\begin{array}{l}\text { modellgestützt, zweiseitige } \\
\text { Märkte }\end{array}$ & $+/-$ & k.A. & $+/-$ \\
\hline Kocsis \& de Bijl (2007) & theoriegeleitet & $+/-$ & $+/-$ & - \\
\hline van Schewick (2007) & theoriegeleitet & - & + & + \\
\hline Economides (2008) & $\begin{array}{l}\text { modellgestützt, zweiseitige } \\
\text { Märkte }\end{array}$ & k.A. & + & + \\
\hline Wallsten \& Hausladen (2009) & $\begin{array}{l}\text { empirisch, } \\
\text { Telekommunikationsmärkte }\end{array}$ & - & k.A. & k.A. \\
\hline Jamison \& Hauge (2008) & $\begin{array}{l}\text { modellgestützt, zweiseitige } \\
\text { Märkte }\end{array}$ & - & - & - \\
\hline $\begin{array}{l}\text { Musacchio, Schwartz \& } \\
\text { Walrand (2009) }\end{array}$ & $\begin{array}{l}\text { modellgestützt, zweiseitige } \\
\text { Märkte }\end{array}$ & $-/+$ & $-/+$ & $-/+$ \\
\hline Litan \& Singer (2007) & theoriegeleitet & - & - & - \\
\hline Renda (2008) & $\begin{array}{l}\text { theoriegeleitet, zweiseitige } \\
\text { Märkte }\end{array}$ & - & k.A. & - \\
\hline Pehnelt (2008) & theoriegeleitet & - & - & - \\
\hline Hermalin \& Katz (2007) & $\begin{array}{l}\text { modellgestützt, zweiseitige } \\
\text { Märkte }\end{array}$ & - & k.A. & k.A. \\
\hline Owen (2007) & theoriegeleitet & - & - & - \\
\hline Brenner et al. (2008) & $\begin{array}{l}\text { theoriegeleitet, zweiseitige } \\
\text { Märkte }\end{array}$ & k.A. & - & k.A. \\
\hline Vogelsang (2007) & theoriegeleitet & - & - & - \\
\hline
\end{tabular}




\section{$4 \quad$ Zusammenfassung und Fazit}

In den vorherigen Abschnitten wurden die Auswirkungen eines nichtneutralen Internets bezüglich Ausschluss, Qualitätsdifferenzierung, Preisdiskriminierung und Innovationswirkungen untersucht. Dabei hat sich gezeigt, dass eine Reihe von positiven Wirkungen eines nichtneutralen Netzes zu erwarten ist. Hierzu zählen die positiven Auswirkungen der Einführung verschiedener Servicequalitäten, die Lösung von Stauproblemen durch Priorisierung der Inhalte und die Steigerung der Wohlfahrt durch Preisdiskriminierung. Ausnahmen, in denen wohlfahrtsminderndes und wettbewerbsschädigendes Verhalten vorliegen könnte, sind ebenso denkbar, betreffen aber vor allem vertikal integrierte und marktmächtige Internet Provider.

Marktbeherrschende Unternehmen unterliegen aber sowohl nach deutschem als auch nach europäischem und US-amerikanischem Wettbewerbsrecht der Missbrauchsaufsicht, sodass mögliches wettbewerbsbeschränkendes Verhalten untersagt werden kann. Des Weiteren besteht das Instrument der ex ante Regulierung monopolistischer Infrastrukturanbieter und Netzbetreiber. Die Empfehlung für die europäische Wettbewerbspolitik lautet daher, sich auf die vorhandene ex post Missbrauchsaufsicht zu beschränken oder aber auf den existierenden Rahmen einer ex ante Regulierung zurückzugreifen. Beide Instrumente erscheinen vollkommen angemessen, um mögliche negative Effekte der Nichtneutralität zu verhindern. Weitere Maßnahmen, die schädliche Auswirkungen einer Nichtneutralität verhindern könnten, wären z.B. durch Informationspflichten zu erreichen (vgl. Vogelsang, 2007 und Dewenter, 2008).

Ein Bereich, der jedoch bisher nicht abschließend beurteilt werden kann, ist der der Innovationswirkungen. Aus den hier vorgestellten Studien lassen sich allerdings keine eindeutigen Effekte herauslesen, die kategorisch für die Notwendigkeit eines neutralen Internets sprechen. Auch wenn hier noch ein großer Forschungsbedarf vorhanden ist, scheinen die Argumente für eine Regulierung von Neutralität auch im Bereich der Innovationen nicht ausreichend. Es ist tendenziell eher davon auszugehen, dass ein Verzicht der Regulierung von Neutralität insgesamt zu einem Anstieg der Innovationstätigkeit führen würde.

Insgesamt liegen also nur wenige und zudem auch wenig überzeugende Argumente vor, die eine regulatorische Festschreibung der Netzneutralität aus ökonomischer Sicht sinnvoll erscheinen lassen. Das existierende Wettbewerbsrecht sollte ausreichend sein, einem Missbrauch einer marktbeherrschenden Stellung vorzubeugen. Auch die zu erwartenden Auswirkungen auf die Innovationstätigkeit der Unternehmen lassen bisher 
keinen dringenden Handlungsbedarf erkennen. Eine Neutralitätsregulierung ist also nach den bisherigen Erkenntnissen nicht angebracht.

\section{Literatur}

Brenner, W., R. Zarnekow, J. Kruse \& A. Sidler (2008), Qualität im Internet. Elektronik und Informationstechnik 125/7/8, 268-273.

Cañón, C. (2009), Regulation Effects on Investment Decisions in TwoSided Market Industries: The Net Neutrality Debate. February 2009, verfügbar unter: http://ssrn.com/abstract=1374782.

Casadesus-Masanell, R., B. Nalebuff \& D. Yoffee (2006), Competing Complements, Working Paper.

Cheng, H., S. Bandyopadhyay \& H. Guo (2007), The Debate on Net Neutrality: A Policy Perspective, Mimeo, University of Florida, verfügbar unter: http://ssrn.com/abstract=959944.

Chen, M.K. \& B. Nalebuff (2006), One-Way Essential Complements, Cowless Foundation Discussion Papers No. 1588, Cowless Foundation for Research in Economics, Yale University, New Haven.

Choi, J.P. and B.C. Kim (2008), Net Neutrality and Investment Incentives, September 2008 CESifo Working Paper Series No. 2390, verfügbar unter: http://ssrn.com/abstract=1264934.

Dewenter, R. (2008), Netzneutralität, in: J. Haucap und J. Kühling (Hrsg.), Effiziente Regeln für Telekommunikationsmärkte in der Zukunft, Kartellrecht, Netzneutralität und Preis-Kosten-Schere, NomosVerlag, Baden-Baden.

Economides, N. (2008), Net Neutrality, Non-Discrimination and Digital Distribution of Content Through the Internet. Journal of Law and Policy for the Information Society 4(2), 209-233.

Economides, N. \& J. Tåg (2008), Net Neutrality on the Internet: A twosided Market Analysis, NET Institute Working Paper No. 07-45, NYU Law and Economics Research Paper 07-40.

Edgeworth, F. (1927), Differential Pricing in a Regime of Competition. Papers Relating to Political Economy 1, 100-107.

Farrell, J. \& M. Katz (2000), Innovation, Rent Extraction, and Integration in Systems Markets. Journal of Industrial Economics 48(4), 413-432. 
Ganley, P. \& B. Allgrove (2006), Net Neutrality: A user's guide. Computer Law \& Security Report 22, 454-463.

Hahn, R.W. \& S. Wallsten (2006), The Economics of Net Neutrality, Discussion Paper, AEI-BROOKINGS Joint Center for Regulatory Studies.

Hausman, J.A. \& J.K. MacKie-Mason (1988), Price Discrimination and Patent Policy. RAND Journal of Economics 19, 253-256.

Hermalin, B. E. \& M. L. Katz (2007), The Economics of Product-Line Restrictions with an Application to the Network Neutrality DebateFebruary 2007. AEI-Brookings Joint Center Working Paper No. 0702, verfügbar unter: http://ssrn.com/abstract=1003391.

Jamison, M. A. \& J.A. Hauge (2008), Getting What You Pay For: Analyzing the Net Neutrality Debate, Public Utility Research Center Working Paper.

Kruse, J. (2007), Internet - Servicequalität oder Netzneutralität, HelmutSchmidt-Universität Hamburg, Mimeo.

Kocsis, V. \& P.W. de Bijl (2007), Network Neutrality and Competition between Networks: A Brief Sketch of the Issues. International Economics and Economic Policy 4(2), 159-184.

Litan, R.E. \& H.J. Singer (2007), Unintended Consequences of Net Neutrality Regulation. Journal on Telecommunications and High Technology Law, Verfügbar unter: http://ssrn.com/abstract=942043.

Musacchio, J., G. Schwartz \& J. Walrand (2009), Network Neutralitiy and Provider Investment Inventives. Review of Network Economics 8(1), S. 22-38.

Owen, B. M. (2007), The Net Neutrality Debate: Twenty Five Years after United States v. AT\&T and 120 Years after the Act of Regulate Commerce.

Pehnelt, G. (2008), The Economics of Net Neutrality Revisited, Jena Economic Research Papers in Economics 2008-080.

Pigou, A.C. (1938), Discriminating Monopoly, in: The Economics of Welfare, 4. Ausgabe, Macmillan \& Co., London.

Ramsey, F.P. (1927), A Contribution to the Theory of Taxation. Economic Journal 37(145), 47-61.

Renda, A. (2008), I Own The Pipes, You Call The Tune - The Net Neutrality Debate And Its (Ir)Relevance For Europe. Center for European Policy Studies Special Report. 
Rochet, J.-C. \& J. Tirole (2003), Platform Competition in Two-Sided Markets. Journal of the European Economic Association 1(4), 9901029.

Sidak, G. (2006), A Consumer-Welfare Approach to Network Neutrality Regulation of the Internet. Journal of Competition Law \& Economics 2(3), 349-474.

van Schewick, B. (2007), Towards an Economic Framework for Network Neutrality Regulation. Journal on Telecommunications and High Technology Law 5, 329-391.

Varian, H. (1988), Price Discrimination, in: R. Schmalensee \& R. Willig (Hrsg.), Handbook of Industrial Organization, Vol. 1, North Holland, Amsterdam.

Vogelsang, I. (2007), Infrastrukturwettbewerb und Netzneutralität, in: A. Picot und A. Freyberg (Hrsg.), Infrastruktur und Services - Das Ende einer Verbindung?, Springer.

Wallsten, S. \& S. Hausladen (2009), Net Neutrality, Unbundling, and their Effects on International Investment in Next-Generation Networks. Review of Network Economics 8(1), 90-112. 


\section{Diskussionspapiere aus dem Institut für Volkswirtschaftslehre der Technischen Universität Ilmenau}

Nr. 15 Kallfass, Hermann H.: Vertikale Verträge und die europäische Wettbewerbspolitik, Oktober 1998. In veränderter Fassung erschienen als: „Vertikale Verträge in der Wettbewerbspolitik der EU“, in: Wirtschaft und Wettbewerb, 49. Jg., 1999, S. 225-244.

Nr. 16 Steinrücken, Torsten: Wirtschaftspolitik für offene Kommunikationssysteme Eine ökonomische Analyse am Beispiel des Internet, März 1999.

Nr. 17 Kallfass, Hermann H.: Strukturwandel im staatlichen Einfluss, April 1999.

Nr. 18 Czygan, Marco: Wohin kann Wettbewerb im Hörfunk führen? Industrieökonomische Analyse des Hörfunksystems der USA und Vergleich mit Deutschland, Dezember 1999.

Nr. 19 Kuchinke, Björn: Sind vor- und vollstationäre Krankenhausleistungen Vertrauensgüter? Eine Analyse von Informationsasymmetrien und deren Bewältigung, September 2000.

Nr. 20 Steinrücken, Torsten: Der Markt für „politische Zitronen“, Februar 2001.

Nr. 21 Kuchinke, Björn A.: Fallpauschalen als zentrales Finanzierungselement für deutsche Krankenhäuser: Eine Beurteilung aus gesundheitsökonomischer Sicht, Februar 2001.

Nr. 22 Kallfass, Hermann H.: Zahlungsunfähige Unternehmen mit irreversiblen Kosten, ihre Fortführungs- und Liquidationswerte, März 2001.

Nr. 23 Kallfass, Hermann H.: Beihilfenkontrolle bei Restrukturierungen und Privatisierungen, April 2001.

Nr. 24 Bielig, Andreas: Property Rights und juristischer Eigentumsbegriff. Leben Ökonomen und Juristen in unterschiedlichen Welten?, Juni 2001.

Nr. 25 Sichelstiel, Gerhard: Theoretische Ansätze zur Erklärung von Ähnlichkeit und Unähnlichkeit in Partnerschaften, Juni 2001.

Nr. 26 Bielig, Andreas: Der „Markt für Naturschutzdienstleistungen“. Vertragsnaturschutz auf dem Prüfstand, Juli 2001.

Nr. 27 Bielig, Andreas: Netzeffekte und soziale Gruppenbildung, Januar 2002.

Nr. 28 Kuchinke, Björn A.; Schubert, Jens M.: Europarechtswidrige Beihilfen für öffentliche Krankenhäuser in Deutschland, April 2002.

Nr. 29 Bielig, Andreas: Messung von Nachhaltigkeit durch Nachhaltigkeitsindikatoren, Februar 2003. 
Nr. 30 Steinrücken, Torsten: Die Legitimation staatlicher Aktivität durch vertragstheoretische Argumente: Anmerkungen zur Kritik an der Theorie des Gesellschaftsvertrages, März 2003.

Nr. 31 Steinrücken, Torsten; Jaenichen, Sebastian: Heterogene Standortqualitäten und Signalstrategien: Ansiedlungsprämien, Werbung und kommunale Leuchtturmpolitik, April 2003.

Nr. 32 Steinrücken, Torsten: Funktioniert ,fairer’ Handel? Ökonomische Überlegungen zum alternativen Handel mit Kaffee, Juli 2003.

Nr. 33 Steinrücken, Torsten; Jaenichen, Sebastian: Die Wiederentdeckung der Zweitwohnsitzsteuer durch die Kommunen - zu Wirkungen und Legitimation aus ökonomischer Sicht, September 2003.

Nr. 34 Rissiek, Jörg; Kressel, Joachim: New Purchasing \& Supply Chain Strategies in the Maintenance, Repair and Overhaul Industry for Commercial Aircraft, September 2003.

Nr. 35 Steinrücken, Torsten; Jaenichen, Sebastian: Europäische Beihilfekontrolle und Public Utilities - Eine Analyse am Beispiel öffentlicher Vorleistungen für den Luftverkehr, Dezember 2003.

Nr. 36 Voigt, Eva; GET UP: Gründungsbereitschaft und Gründungsqualifizierung Ergebnisse der Studentenbefragung an der TU Ilmenau, April 2004.

Nr. 37 Steinrücken, Torsten; Jaenichen, Sebastian: Levelling the playing field durch staatliche Beihilfen bei differierender Unternehmensmobilität, Mai 2004.

Nr. 38 Steinrücken, Torsten; Jaenichen, Sebastian: Sekundärwirkungen von Unternehmensansiedlungen - Eine Beurteilung staatlicher Aktivität beim Auftreten paretorelevanter Nettoexternalitäten, Juni 2004.

Nr. 39 Kallfaß, Hermann H.: Wettbewerb auf Märkten für Krankenhausdienstleistungen - eine kritische Bestandsaufnahme, Juni 2004.

Nr. 40 Engelmann, Sabine: Internationale Transfers und wohlfahrtsminderndes Wachstum, September 2004.

Nr. 41 Steinrücken, Torsten; Jaenichen, Sebastian: Zum Einfluss von Ausländern auf die Wirtschaftsleistung von Standorten - Ist Zuwanderung ein Weg aus der ostdeutschen Lethargie?, Oktober 2004.

Nr. 42 Steinrücken, Torsten; Jaenichen, Sebastian: Wer ist wirklich reich? - Zu Problemen der Wohlfahrtsmessung durch das Bruttoinlandsprodukt, April 2005.

Nr. 43 Steinrücken, Torsten; Jaenichen, Sebastian: Wo bleiben die Subventionssteuern? - Probleme des Beihilfenrechts und ein alternatives Regulierungskonzept, Mai 2005. 
Nr. 44 Jaenichen, Sebastian; Steinrücken, Torsten; Schneider, Lutz: Zu den ökonomischen Wirkungen gesetzlicher Feiertage - Eine Diskussion unter besonderer Berücksichtigung der Arbeitszeitpolitik, Juni 2005.

Nr. 45 Kuchinke, Björn A.: Qualitätswettbewerb zwischen deutschen Akutkrankenhäusern unter besonderer Berücksichtigung von DRG und Budgets, Juni 2005.

Nr. 46 Kuchinke, Björn A.; Walterscheid, Heike: Wo steht der Osten? Eine ökonomische Analyse anhand von Wohlfahrts- und Happinessindikatoren, Juni 2005.

Nr. 47 Kuchinke, Björn A.; Schubert, Jens M.: Staatliche Zahlungen an Krankenhäuser: Eine juristische und ökonomische Einschätzung nach Altmark Trans und der Entscheidung der Kommission vom 13.7.2005, August 2005.

Nr. 48 Steinrücken, Torsten; Jaenichen, Sebastian: Überkapazitäten zur Absicherung politischer Risiken und Instrumente finanzwirtschaftlicher Gegensteuerung, November 2005.

Nr. 49 Jaenichen, Sebastian; Steinrücken, Torsten: Opel, Thüringen und das Kaspische Meer, Januar 2006.

Nr. 50 Kallfaß, Hermann H.: Räumlicher Wettbewerb zwischen Allgemeinen Krankenhäusern, Februar 2006.

Nr. 51 Sickmann, Jörn: Airport Slot Allocation, März 2006.

Nr. 52 Kallfaß, Hermann H.; Kuchinke, Björn A.: Die räumliche Marktabgrenzung bei Zusammenschlüssen von Krankenhäusern in den USA und in Deutschland: Eine wettbewerbsökonomische Analyse, April 2006.

Nr. 53 Bamberger, Eva; Bielig, Andreas: Mehr Beschäftigung mittels weniger Kündigungsschutz? Ökonomische Analyse der Vereinbarungen des Koalitionsvertrages vom 11. 11. 2005, Juni 2006.

Nr. 54 Jaenichen, Sebastian; Steinrücken, Torsten: Zur Ökonomik von Steuergeschenken - Der Zeitverlauf als Erklärungsansatz für die effektive steuerliche Belastung, Dezember 2006.

Nr. 55 Jaenichen, Sebastian; Steinrücken, Torsten: Wirkt eine Preisregulierung nur auf den Preis? Anmerkungen zu den Wirkungen einer Preisregulierung auf das Werbevolumen, Mai 2007.

Nr. 56 Kuchinke, B. A.; Sauerland, D.; Wübker, A.: Determinanten der Wartezeit auf einen Behandlungstermin in deutschen Krankenhäusern - Ergebnisse einer Auswertung neuer Daten, Februar 2008.

Nr. 57 Wegehenkel, Lothar; Walterscheid, Heike: Rechtsstruktur und Evolution von Wirtschaftssystemen - Pfadabhängigkeit in Richtung Zentralisierung?, Februar 2008.

Nr. 58 Steinrücken, Torsten; Jaenichen, Sebastian: Regulierung und Wohlfahrt in einem Modell mit zwei Aktionsparametern, März 2008. 
Nr. 59 Lehnert, Ninja M.: Externe Kosten des Luftverkehrs - Ein Überblick über den aktuellen Stand der Diskussion, April 2008.

Nr.60 Walterscheid, Heike: Reformbedarf etablierter Demokratien im Kontext dezentralisierter Gesellschaftssysteme - Grundlegende Hindernisse bei Steuersystemreformen“, ... 2008.

Nr. 61 Walterscheid, Heike; Wegehenkel, Lothar: Kostenstruktur, Zahlungsbereitschaft und das Angebot von Mediengütern auf Medienmärkten, Juni 2008.

Nr.62 Walterscheid, Heike; Wegehenkel, Lothar: Wohlstand der Nationen und handlungsrechtliche Struktur eines Gesellschaftssystems, September 2008.

Nr. 63 Dewenter, Ralf; Haucap, Justus; Wenzel, Tobias: Indirect Network Effects with Two Salop Circles: The Example oft the Music Industry, Juni 2009.

Nr.64 Dewenter, Ralf; Jaschinski, Thomas; Wiese, Nadine: Wettbewerbliche Auswirkungen eines nichtneutralen Internets, Juli 2009. 